\title{
Dynamic stability of the Solar System: Statistically inconclusive results from ensemble integrations
}

\author{
Richard E. Zeebe ${ }^{1, *}$ \\ * Corresponding Author. \\ ${ }^{1}$ School of Ocean and Earth Science and Technology, University of Hawaii at Manoa, 1000 Pope Road, \\ MSB 629, Honolulu, HI 96822, USA. email: zeebe@soest.hawaii.edu
}

The Astrophysical Journal, accepted, Oct 08, 2014

\begin{abstract}
Due to the chaotic nature of the Solar System, the question of its long-term stability can only be answered in a statistical sense, for instance, based on numerical ensemble integrations of nearby orbits. Destabilization of the inner planets, leading to close encounters and/or collisions can be initiated through a large increase in Mercury's eccentricity, with a currently assumed likelihood of $\sim 1 \%$. However, little is known at present about the robustness of this number. Here I report ensemble integrations of the full equations of motion of the eight planets and Pluto over 5 Gyr, including contributions from general relativity. The results show that different numerical algorithms lead to statistically different results for the evolution of Mercury's eccentricity $\left(e_{\mathcal{M}}\right)$. For instance, starting at present initial conditions $\left(e_{\mathcal{M}} \simeq 0.21\right)$, Mercury's maximum eccentricity achieved over 5 Gyr is on average significantly higher in symplectic ensemble integrations using heliocentric than Jacobi coordinates and stricter error control. In contrast, starting at a possible future configuration $\left(e_{\mathcal{M}} \simeq 0.53\right)$, Mercury's maximum eccentricity achieved over the subsequent $500 \mathrm{Myr}$ is on average significantly lower using heliocentric than Jacobi coordinates. For example, the probability for $e_{\mathcal{M}}$ to increase beyond 0.53 over $500 \mathrm{Myr}$ is $>90 \%$ (Jacobi) vs. only $40-55 \%$ (heliocentric). This poses a dilemma as the physical evolution of the real system — and its probabilistic behavior - cannot depend on the coordinate system or numerical algorithm chosen to describe it. Some tests of the numerical algorithms suggest that symplectic integrators using heliocentric coordinates underestimate the odds for destabilization of Mercury's orbit at high initial $e_{\mathcal{M}}$.
\end{abstract}

Subject headings: celestial mechanics - methods: numerical — methods: statistical — planets and satellites: dynamical evolution and stability

\section{Introduction}

The question whether the Solar System is dynamically stable over long periods of time has received considerable attention for centuries, including contributions from Newton, Lagrange, Laplace, Poincaré, Kolmogorov, Arnold, Moser etc. (e.g. Laskar 2013). Research in this field has recently experienced a renaissance due to advances in computational power and numerical al- gorithms, permitting integration of the full equations of motion approaching the Solar System's lifetime $( \pm \sim 5$ Gyr) (Wisdom \& Holman 1991; Quinn et al. 1991; Sussman \& Wisdom 1992; Saha \& Tremaine 1992; Murray \& Holman 1999; Ito \& Tanikawa 2002; Varadi et al. 2003; Batygin \& Laughlin 2008; Laskar \& Gastineau 2009). Moreover, parallel computing now allows tackling the problem of stability with statistical means through simultaneous integration of multiple nearby orbits 
(Laskar \& Gastineau 2009). A statistical approach is necessary because of the chaotic behavior of the system, i.e. the sensitivity of orbital solutions to initial conditions (Laskar 1989; Sussman \& Wisdom 1992; Murray \& Holman 1999; Richter 2001; Varadi et al. 2003; Batygin \& Laughlin 2008). Small differences in initial conditions grow exponentially, with a time constant (Lyapunov time) for the inner planets of only 5 Myr (Laskar 1989; Varadi et al. 2003; Batygin \& Laughlin 2008). For example, a difference in initial coordinates of $1 \mathrm{~mm}$ grows to $\sim 1 \mathrm{AU}\left(=1.496 \times 10^{11} \mathrm{~m}\right)$ after $163 \mathrm{Myr}$. Thus, the chaotic nature of the planetary orbits makes it fundamentally impossible to predict their evolution accurately beyond $\sim 100$ Myr (Laskar 1989). Hence the stability problem can only be answered in probabilistic terms, e.g. by studying the behavior of a large number of physically possible solutions. The quest for a single deterministic solution, which conclusively describes the Solar System's evolution for all times (in the spirit of Laplace's demon, Laplace 1951), must be regarded as quixotic.

An ensemble integration of 2,501 nearby orbits over 5 Gyr has recently been reported based on the full equations of motion and including contributions from general relativity and the Moon (Laskar \& Gastineau 2009). In those simulations, two adjacent orbits differed initially by only $0.38 \mathrm{~mm}$ in Mercury's semi-major axis $\left(a_{\mathcal{M}}\right)$. The largest overall offset in initial $a_{\mathcal{M}}$ was hence $2,500 \times 0.38 \mathrm{~mm}=0.95 \mathrm{~m}$, well within the uncertainty of our current knowledge of the Solar System. In about $1 \%$ of the simulations, a large increase in Mercury's eccentricity was reported, which can lead to destabilization of the inner planets, including close encounters and/or collisions. Note that the obtained probability distribution of Mercury's eccentricity was similar to results obtained earlier with averaged equations (Laskar 2008). Most surprisingly, further simulations included the possibility of a collision between Earth and Venus via transfer of angular momentum from the giant planets to the terrestrial planets (Laskar \& Gastineau 2009). Given the chaotic behavior of the system, such an outcome might be within the range of possibilities. However, little is currently known about the potential dependence of the simulated trajectories and statistical results on the numerical integrator, step size, integration coordinates, etc.

Here I report ensemble integrations of Solar System orbits over 5 Gyr, including contributions from general relativity. The simulations reveal a strong influence of integration coordinates on statistical results, i.e. on the predicted odds for destabilization of Mercury's orbit. Furthermore, I discuss resonances and Lyapunov times, and perform several tests aiming at resolving the dilemma of the dependency of statistical results on integration coordinates.

\section{Methods}

The full equations of motion of the eight planets and Pluto were integrated over 5 Gyr into the future using the numerical integrator packages mercury6 and HNBody and various integration options (Chambers 1999; Rauch \& Hamilton 2002). Unless stated otherwise, symplectic integrators were used. Symplectic integrators exactly describe the time evolution of a (modified) Hamiltonian system that is very close to the true Hamiltonian (cf. e.g. Wisdom \& Holman 1991; Yoshida 1993; Chambers 1999) and hence do not suffer from long-term buildup in energy error. The maximum energy variation along a given orbit in symplectic integrations (see below) provides a measure of the difference between the modified and the true Hamiltonian. Relativistic corrections are critical (Laskar \& Gastineau 2009) and were available in HNBody but not in mercury6. PostNewtonian corrections for symplectic integration (Mikkola 1998; Soffel 1989) were therefore implemented before using mercury6 (see Appendix). Thus, all simulations presented here include contributions from general relativity, unless explicitly stated. To allow comparison with previous studies, several higher-order effects were not included here. This means ignoring potential effects from asteroids (Ito \& Tanikawa 2002; Batygin \& Laughlin 2008), perturbations from passing stars, and solar mass loss (Ito \& Tanikawa 2002; Batygin \& Laughlin 2008; Laskar \& Gastineau 2009). Furthermore, the Earth-Moon system was considered a single mass point, located at the Earth-Moon barycenter.

Per ensemble integration, 40 orbital solutions were computed with each package, starting from the same set of initial conditions, where Mercury's 
initial radial distance was offset by $7 \mathrm{~cm}$ between every two adjacent orbits (largest overall offset: $39 \times 7 \mathrm{~cm}=2.73 \mathrm{~m}$ ). Initial conditions for all bodies in the 5-Gyr runs (before offsetting Mercury) were generated from DE431 (naif.jpl.nasa. gov/pub/naif/generic_kernels/spk/planets) at JD2451544.5 (01 Jan 2000) using the SPICE toolkit for Matlab (naif.jpl.nasa.gov/naif/ toolkit.html) (Table 2).

For the 5-Gyr simulations with mercury6, the hybrid integrator with democratic-heliocentric coordinates (Chambers 1999; Duncan et al. 1998) following Batygin \& Laughlin (2008) and a 6-day initial timestep $(\Delta t)$ for the 2 nd order symplectic algorithm was used. In case Mercury's eccentricity $\left(e_{\mathcal{M}}\right)$ increased above certain threshold values during the 5-Gyr simulations with mercury6, $\Delta t$ was reduced but held constant after that until the next threshold was crossed (if applicable). For example, starting at $\Delta t=6$ days and $e_{\mathcal{M}}=0.21, \Delta t$ was reduced to 5 days when $e_{\mathcal{M}}$ exceeded 0.34. mercury6's source code is available (Chambers 1999) and was manipulated accordingly. The threshold values and corresponding timesteps (Table 1) were chosen to maintain a roughly similar maximum relative energy error $\left(\max |\Delta E / E|=\max \left|\left(E(t)-E_{0}\right) / E_{0}\right|\right)$ throughout the simulation. HNBody's source code is not available (Rauch \& Hamilton 2002) and a constant 4day timestep was used throughout the 5-Gyr simulations, employing a 2nd order symplectic integrator with corrector and Jacobi coordinates (see below and Wisdom \& Holman 1991).

Table 1: Threshold $e_{\mathcal{M}}$ values and timesteps for the 5-Gyr simulations with mercury6.

\begin{tabular}{lrrrrrr}
\hline \hline$e_{\mathcal{M}}>$ & 0.34 & 0.38 & 0.44 & 0.49 & 0.57 & 0.62 \\
\hline$\Delta t(\mathrm{~d})$ & 5 & 4 & 3 & 2 & 1.5 & 1 \\
\hline
\end{tabular}

The 40 orbital solutions were simultaneously integrated on a 64-bit Linux cluster (one 5-Gyr job per core on 10 Intel i7-3770 $3.40 \mathrm{GHz}$ nodes with 4 cores each). Typical integration times for the 5-Gyr runs with mercury6 were $12-23$ days wall-clock time depending on step-size reduction ( $\Delta t \leq 6 \mathrm{~d}$, see above) and 12 days wall-clock time with HNBody $(\Delta t=4 \mathrm{~d})$. Integrations with HNBody were performed using the pre-compiled binary ver- sion for Linux 64-bit Intel/AMD64. Executables of mercury6 were compiled from source code on 64-bit Linux platforms using gfortran 4.6.3. When compared to test runs with mercury 6 on a true 32-bit machine, a small long-term decrease in angular momentum was noticeable in the output of the 64-bit mercury6 executable at small step size. The issue disappeared when using one of the following options on 64-bit platforms. (1) Compile with flag -m32 (generates code for a 32-bit environment but is slower). (2) In the mercury6 subroutine drift_kepmd ( ) replace the original approximations for $\sin (x)$ and $\cos (x)$ by actual $\sin (x)$ and $\cos (x)$ functions.

\subsection{Democratic-heliocentric- and Jacobi coordinates}

The effect of different integration coordinates on the results of the symplectic integrations will be discussed at length below. Hence the definition of the coordinates is given here. Democraticheliocentric coordinates simply consist of heliocentric (bodycentric) positions and barycentric momenta (Duncan et al. 1998; Chambers 1999), see Section 6.1.

Jacobi coordinates have a slightly more complex structure but are useful in symplectic integrations of the $n$-body problem because they allow writing the kinetic energy as a diagonal sum of squares of the momenta (Wisdom \& Holman 1991). In Jacobi coordinates, the first coordinate, $\boldsymbol{Q}_{0}$, may be taken as the position of the center of mass. The first relative coordinate is the position of the first planet relative to the central body. The second relative coordinate is the position of the second planet relative to the center of mass of the central body and the first planet, and so forth. Thus, the $i$ th relative coordinate is the position of the $i$ th planet relative to the center of mass of the central body and the planets with lower indices:

$$
\boldsymbol{Q}_{i}=\boldsymbol{x}_{i}-\boldsymbol{X}_{i-1},
$$

where $0<i<n$ and:

$$
\boldsymbol{X}_{i}=M_{i}^{-1} \sum_{j=0}^{i} m_{j} \boldsymbol{x}_{j} \quad ; \quad M_{i}=\sum_{j=0}^{i} m_{j}
$$

is the center of mass of bodies with indices up to $i$. The momenta are:

$$
\boldsymbol{P}_{i}=m_{i}^{\prime} \boldsymbol{V}_{i},
$$


where $\boldsymbol{V}_{i}$ is the time derivative of $\boldsymbol{Q}_{i}$ and $m_{i}^{\prime}=$ $M_{i-1} m_{i} / M_{i}$ for $0<i<n$ and $m_{0}^{\prime}=M_{n-1}=M$, is the sum of all masses. Interestingly, because of the Sun's dominant mass, the difference in the actual values of heliocentric and Jacobi coordinates is small in the Solar System (e.g. zero for Mercury and of order $10^{-7}$ and $10^{-6}$ AU for Venus and Earth). Yet the difference in the results of symplectic integrations due to these different sets of coordinates is significant (see below). Note that the comment above is meant for illustrative purposes only. All initial input coordinates for mercury6 and HNBody were specified in heliocentric positions and velocities (e.g. Table 2). Transformation to the desired set of integration coordinates is performed internally. In the following, "same initial conditions" refers to truly identical initial conditions, regardless of the integration coordinates used.

\section{Results: 5-Gyr and 500-Myr simula- tions}

At first glance, the 5-Gyr simulations with mercury 6 and HNBody appeared to yield similar results, except for different energy and momentum errors (Fig. 1). In none of the 80 simulations a large increase in $e_{\mathcal{M}}$ or a destabilization of the inner solar system occurred, which is also not expected if the corresponding odds are $\sim 1 \%$ (Laskar \& Gastineau 2009). However, at second glance differences between the mercury 6 and HNBody runs became apparent. For runs with identical initial conditions, the maximum difference in Mercury's eccentricity $\left(\Delta e_{\mathcal{M}}\right)$ between the mercury 6 and HNBody simulations typically grew from $\sim 10^{-7}$ to 0.01 over only $\sim 10 \mathrm{Myr}$. For comparison, when HNBody was run with bodycentric coordinates (all else equal), $\Delta e_{\mathcal{M}}$ grew to 0.01 over $\sim 60 \mathrm{Myr}$ (see below). Furthermore, $\Delta e_{\mathcal{M}}$ between two simulations with the same package and options but different initial conditions grew to 0.01 over $\sim 75 \mathrm{Myr}$ and $\sim 130 \mathrm{Myr}$, respectively, for the above mercury6 and HNBody setup (Fig. 5). While the divergence of trajectories after $\sim 60$ Myr may be attributed to the chaotic behavior of the physical system (Laskar 1989; Varadi et al. 2003; Batygin \& Laughlin 2008), the rapid rise in $\Delta e_{\mathcal{M}}$ over only $\sim 10 \mathrm{Myr}$ between the 5 Gyr runs with democratic-heliocentric (mercury6) and Jacobi coordinates (HNBody) hints to a po-
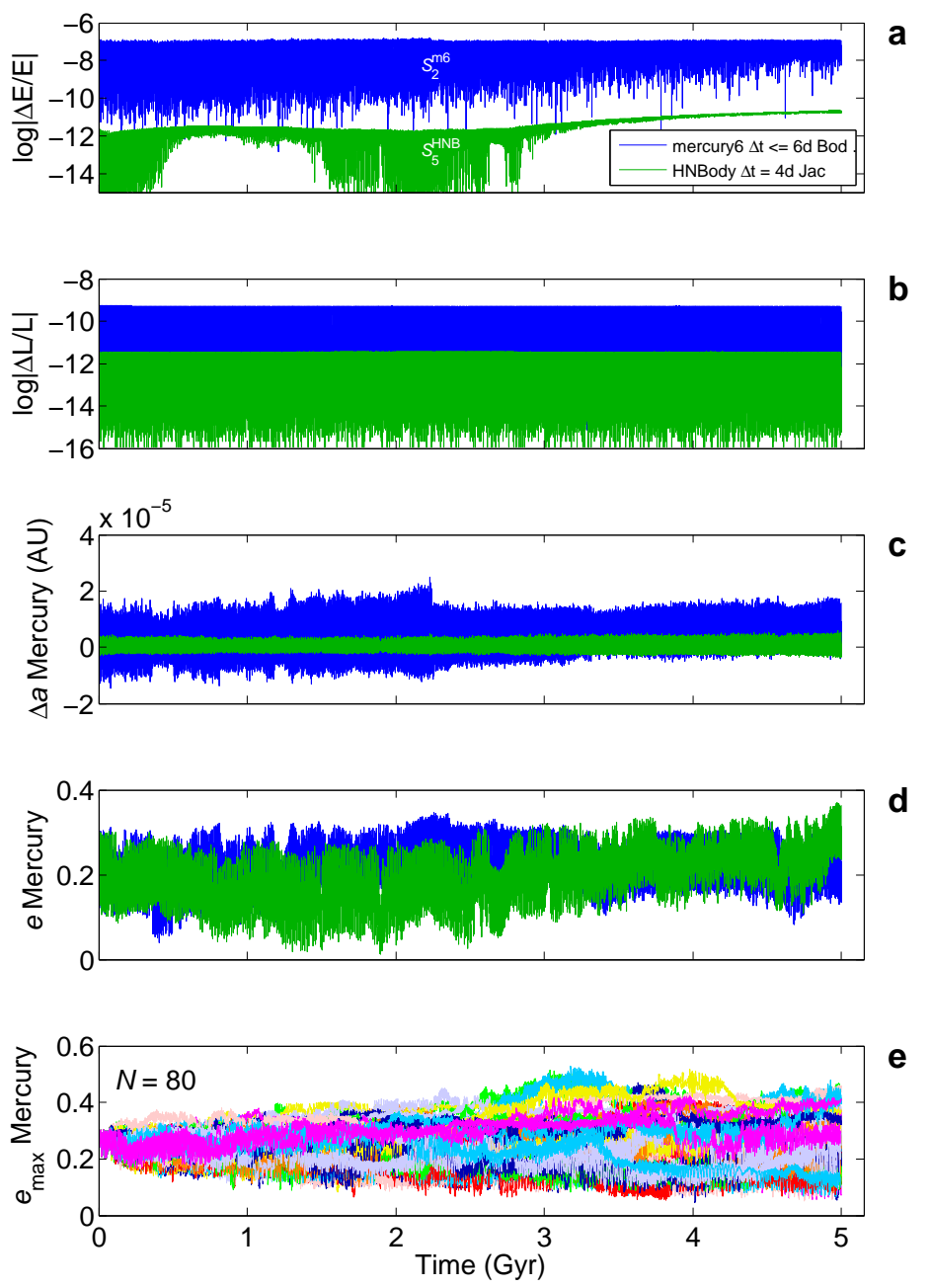

Fig. 1.- Results from 5-Gyr runs with mercury6 and HNBody. Blue curves (a) - (d): mercury6, run \#2 (solution $\left.S_{2}^{\mathrm{m} 6}\right), \Delta t \leq 6$ days, democratic-heliocentric coordinates. Green curves (a)-(d): HNBody run $\# 5\left(S_{5}^{\mathrm{HNB}}\right)$, $\Delta t=4$ days, Jacobi coordinates. (a) Relative energy error, $|\Delta E / E|=\left|\left(E(t)-E_{0}\right) / E_{0}\right|$. (b) Relative angular momentum error, $|\Delta L / L|=\left|\left(L(t)-L_{0}\right) / L_{0}\right|$. (c) Change in Mercury's semi-major axis, $\Delta a=a(t)-a_{0}$. (d) Mercury's eccentricity $\left(e_{M}\right)$. (e) Mercury's maximum eccentricity (per 1 Myr bin) from all 5-Gyr runs $(N=80)$ with mercury6 and HNBody $(N=40$ each). Note time step reduction from 6 to 5 days at $\sim 2.24$ Gyr in $S_{2}^{\mathrm{m} 6}$ (see (a) and (c)).

tential numerical origin. A rapid $\Delta e_{\mathcal{M}}$ rise over $\sim 10$ Myr was also observed between two HNBody runs with identical initial conditions and timestep but bodycentric vs. Jacobi coordinates. In addition, orbital solutions obtained with bodycentric and Jacobi coordinates yield different Lyapunov times for the inner planets (see below).

The maximum in the relative energy error in 
the 5-Gyr mercury6 runs was typically $10^{4}$ times larger than in HNBody; the corresponding maximum angular momentum error about $10^{2}$ times larger (Fig. 1). One might hence be tempted to argue that any differences between the present 5Gyr simulations are due to larger errors in the mercury6 simulations (less reliable), rather than heliocentric vs. Jacobi coordinates. However, this argument is not supported by the results of additional ensemble simulations (see below). The small offsets in Mercury's initial radial distance randomized the initial conditions and led to complete divergence of eccentricities after $\sim 100 \mathrm{Myr}$ (Fig. 1). No patterns in the evolution of Mercury's eccentricity were observed between adjacent orbits (or sets of orbits); neither appeared pairs of mercury 6 and HNBody runs starting from identical initial conditions lead to correlations in the behavior of $e_{\mathcal{M}}$ over 5 Gyr.

However, statistically the 5-Gyr ensemble simulations with mercury6 (heliocentric coordinates) and HNBody (Jacobi coordinates) led to significantly different distributions for the maximum eccentricities of Mercury $\left(e_{\mathcal{M}}^{\max }\right)$ achieved over $5 \mathrm{Gyr}$ ( $N=40$ each, Fig. 2). More precisely, the null hypothesis that the two $e_{\mathcal{M}}^{\max }$ samples from the 5 -Gyr mercury6 and HNBody ensemble simulations are random samples from normal distributions with equal means can be rejected ( $p<0.017$, two-tailed Student's $t$-test). Furthermore, the probability for $e_{\mathcal{M}}$ to grow, say, beyond 0.4 is more than double for the 5 -Gyr mercury6 setup $(11 / 40=28 \%)$ than for the HNBody setup $(5 / 40=13 \%)$ (Fig. 2).

This statistical discrepancy (obtained at relatively low initial $e_{\mathcal{M}} \simeq 0.21$ ) raises questions about algorithm performance at high $e_{\mathcal{M}}$. For instance, one might expect that $e_{\mathcal{M}}$ also grows larger on average at initially high $e_{\mathcal{M}}$ when using heliocentric vs. Jacobi coordinates. The issue is critical for the potential destabilization of the inner planets as a result of a large increase in Mercury's eccentricity. Thus, I conducted additional ensemble integrations over $500 \mathrm{Myr}$ starting at the time/conditions of the run with the highest $e_{\mathcal{M}} \simeq$ 0.53 (solution $S_{12}^{\mathrm{m} 6}$ ) achieved during the 5-Gyr simulations (Figs. 2 and 3). The timestep was reduced to 2 days in both the mercury 6 and HNBody setup. Surprisingly, in this case, the mercury6 simulations (heliocentric coordinates) gave a significantly smaller mean $e_{\mathcal{M}}^{\max }$ value than the HNBody simula-
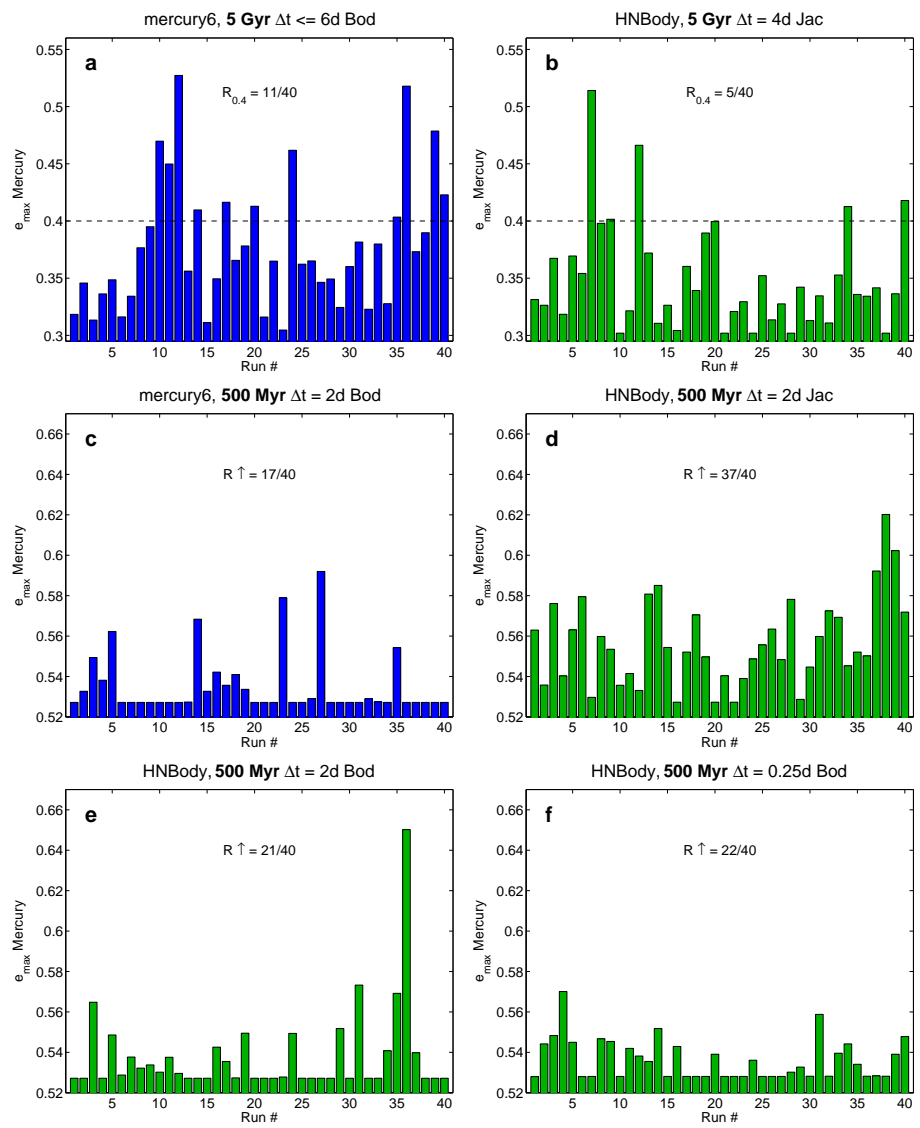

Fig. 2.- Mercury's maximum eccentricity $\left(e^{\max }\right)$ achieved during 5-Gyr and 500-Myr runs with mercury6 (blue bars) and HNBody (green bars). Bod: bodycentric, Jac: Jacobi coordinates. (a) Mean $e_{\mathcal{M}}^{\max }=0.377 . e_{\mathcal{M}}$ grows beyond 0.4 in 11 out of 40 solutions $\left(R_{0.4}=11 / 40=28 \%\right)$. (b) Mean $e_{\mathcal{M}}^{\max }=0.349 ; R_{0.4}=5 / 40=13 \%$. (c) Mean $e_{\mathcal{M}}^{\max }=0.535 ; \quad e_{\mathcal{M}}$ grows beyond start value of 0.53 in 17 out of 40 solutions $\left(R_{\uparrow}=17 / 40=43 \%\right)$. (d) Mean $e_{\mathcal{M}}^{\max }=0.557 ; R_{\uparrow}=37 / 40=93 \%$. (e) Mean $e_{\mathcal{M}}^{\max }=0.538 ; R_{\uparrow}=21 / 40=53 \%$. (f) Mean $e_{\mathcal{M}}^{\max }=0.536$; $R_{\uparrow}=22 / 40=55 \%$. The null hypothesis that two pairwise $e^{\max }$ samples as shown in panels $\alpha=a, c, e, f$ and $\beta=b, d$ $(N=40$ each $)$ are random samples from normal distributions with equal means can be rejected at the following significance levels $p_{\alpha \beta}$ (two-tailed Student's $t$-test): $p_{a b}<0.017$, $p_{c d}<2.2 \times 10^{-6}, p_{e d}<0.0003, p_{f d}<1.3 \times 10^{-6}$.

tions (Jacobi coordinates) $\left(p<2.2 \times 10^{-6}\right)$. Also, the probability for $e_{\mathcal{M}}$ to increase above the start value of 0.53 is less than half for the mercury6 setup $(17 / 40=43 \%)$ than for the HNBody setup $(37 / 40=93 \%)$ (Fig. 2).

This result appears unrelated to the integrator package but related to the choice of the integration coordinates. Using HNBody with bodycentric coordinates for the 500-Myr runs gives a mean $e_{\mathcal{M}}^{\max }$ 


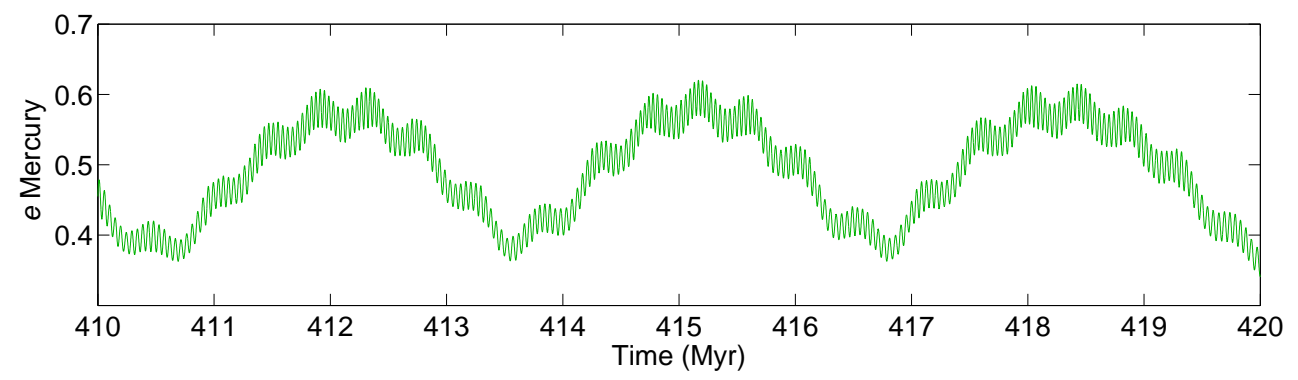

Fig. 3.- Example of Mercury's eccentricity $\left(e_{\mathcal{M}}\right)$ during a 500 -Myr run with HNBody ( $\Delta t=2$ days, Jacobi coordinates). The pattern is typical during $e_{\mathcal{M}}$ increases associated with the $\left(g_{1}-g_{5}\right)$ resonance (see text).

value similar to the heliocentric mercury6 setup and significantly lower than the HNBody setup with Jacobi coordinates $(p<0.0003$, Fig. 2). I also tested whether the statistically different $e_{\mathcal{M}}^{\max }$ at Mercury's higher initial eccentricity is related to typically larger errors associated with bodycentric vs. Jacobi coordinates at the same step size. I repeated the 500-Myr runs with the bodycentric HNBody setup but an eight-fold smaller timestep $(0.25 \mathrm{~d}, \sim 100$-fold smaller $|\Delta E / E|)$. However, the basic result remained the same. At high initial $e_{\mathcal{M}}$, the setup using bodycentric coordinates leads to significantly smaller mean $e_{\mathcal{M}}^{\max }$ than the setup using Jacobi coordinates $\left(p<1.3 \times 10^{-6}\right.$, Fig. 2). This suggests that the statistical discrepancies are due to integration coordinates, not errors in energy or angular momentum (see above).

This poses a quandary because the evolution of the physical Solar System, including its probabilistic behavior, cannot depend on our choice of coordinate system or numerical algorithm. Fundamentally, there is no reason to prefer one set of coordinates over the other. However, symplectic schemes differ in their implementation of different integration coordinates (Section 6.1). While in symplectic algorithms the size of the perturbation term (planet interactions) may be numerically larger in heliocentric than in Jacobi coordinates, little performance difference has been reported over 100,000 steps (Farrés et al. 2013). Furthermore, heliocentric and Jacobi coordinates appear to have opposing effects on $e_{\mathcal{M}}^{\max }$ at low and high initial $e_{\mathcal{M}}$, pointing to a more complex origin. Importantly, differences per timestep due to integration coordinates, which may ultimately cause differences in eccentricity, are minuscule.
For example, in HNBody ( $\Delta t=6$ days, body vs. Jacobi) it takes $\sim 200 \mathrm{kyr}$, or 12 million steps for $\Delta e_{\mathcal{M}}$ to grow from $10^{-5}$ to $10^{-4}(\sim 1$ billion steps for Jupiter). Statistical differences between $e_{\mathcal{M}}^{\max }$ in the 500-Myr runs only become significant $(p<$ 0.05 ) after $\sim 140 \mathrm{Myr}$, or 25 billion steps (HNBody, $\Delta t=2$ days, body vs. Jacobi).

\section{Fourier analysis: $g_{1}-g_{5}$ Resonance}

Fourier analysis of Mercury's longitude of perihelion showed that Mercury's eccentricity increase was generally associated with a shift in eigenfrequency $\left(g_{1}\right)$ towards Jupiter's forcing frequency $\left(g_{5}\right)$. For most 500-Myr runs, a correlation between $e_{\mathcal{M}}^{\max }$ and $g_{1}$ was observed (Fig. 4). This is consistent with the pattern of a secular resonance involving Mercury and Jupiter (plus Venus' participation) (Batygin \& Laughlin 2008; Laskar 2008; Lithwick \& Wu 2011; Boué et al. 2012) and appears to be the common cause for $e_{\mathcal{M}}$ increases in nearly all 500-Myr runs, regardless of integrator package or coordinates. Note that contributions from general relativity (Einstein 1916) as included here (Mikkola 1998; Soffel 1989) are important as they universally move $g_{1}$ up (by $\sim 0.43$ " $\mathrm{y}^{-1}$ at present) and away from the $\left(g_{1}-g_{5}\right)$ resonance, substantially reducing the probability for large $e_{\mathcal{M}}$ increases across all simulations (Laskar 2008; Laskar \& Gastineau 2009) (Fig. 4).

More importantly, in the 500-Myr runs bodycentric coordinates showed on average a higher tendency towards resonance damping and hence larger $g_{1}$ and smaller $e_{\mathcal{M}}^{\max }$, compared to Jacobi coordinates. This tendency is significant and roughly halves the probability for $e_{\mathcal{M}}$ to increase beyond 0.53 in the 500-Myr runs (Fig. 2). The cause for the statistical differences originating 


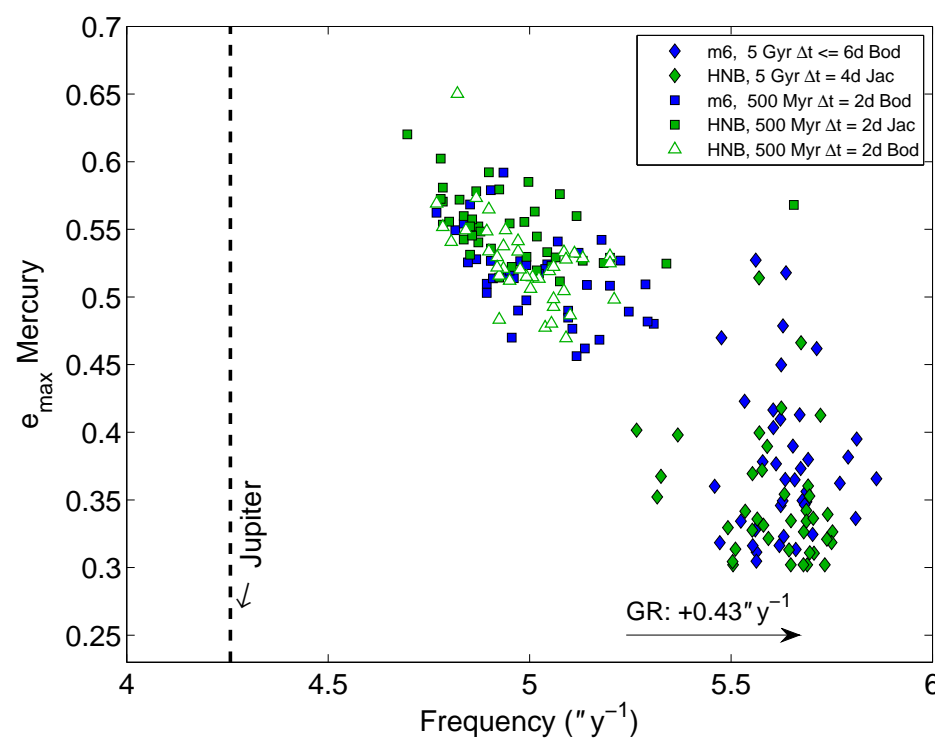

Fig. 4.- Mercury's maximum eccentricity $\left(e_{\mathcal{M}}^{\max }\right)$ vs. eigenfrequency $g_{1}$ in arcsec $\mathrm{y}^{-1}$ (symbols; $g_{1}$ from Fourier analysis of Mercury's longitude of perihelion, $\varpi_{1}$ ). m6: mercury6, HNB: HNBody, Bod: bodycentric, Jac: Jacobi coordinates. Dashed line: Jupiter's forcing frequency. For the 5-Gyr runs, $e_{\mathcal{M}}^{\max }$ and $g_{1}$ values shown were determined using output for $e_{\mathcal{M}}$ and $\varpi_{1}$ over the full 5-Gyr time interval. For the 500-Myr runs, $e_{M}^{\max }$ and $g_{1}$ values shown were determined only over the final 250 Myr interval of the runs. This improves the representation of solutions with long-term decline in $e_{\mathcal{M}}$ below the 0.53 -start value. Otherwise these solutions would all plot at constant $e_{\mathcal{M}}^{\max }=0.53$ despite large variations in $g_{1}$. Contributions from general relativity (included in all simulations) universally move $g_{1}$ up (by $\sim 0.43{ }^{\prime} \mathrm{y}^{-1}$ at present, arrow) and away from the $\left(g_{1}-g_{5}\right)$ resonance.

from different integration coordinates as found here is not obvious, neither is clear which (if any) of the methods provides accurate probability predictions over $10^{8}-10^{9}$-year timescale (see below).

\section{Mercury's eccentricity and Lyapunov times from different simulations}

Starting from slightly different initial conditions $(2.73 \mathrm{~m}$ offset in Mercury's initial radial distance), the computed difference in Mercury's eccentricity $\left(\Delta e_{\mathcal{M}}\right)$ between two solutions grows slowly over the first $50 \mathrm{Myr}$ or more when using the same program and the same integration coordinates (only bodycentric or only Jacobi coordinates, Fig. 5a-e). This is also true for the same initial conditions, different programs (mercury6 vs. HNBody) and same integration coordinates (both bodycentric, Fig. 5f). However, using the same initial conditions but different integration coordi- nates (bodycentric vs. Jacobi coordinates), $\Delta e_{\mathcal{M}}$ between two solutions grows quickly over the first $10 \mathrm{Myr}$ (Fig. 5g-j). This feature was observed irrespective of whether the same or different programs were used, whether general relativity contributions were included or not, and whether the initial eccentricity was low or high (initial $e_{\mathcal{M}}=$ $0.21 / 0.53$, see above).

During the slow initial rise (Fig. $5 \mathrm{a}-\mathrm{f}$ ), $\Delta e_{\mathcal{M}}$ is usually dominated by polynomial growth and may increase linearly with time in a log-log plot (Laskar 1989; Varadi et al. 2003). The subsequent rapid rise after $\gtrsim 50$ Myr until $\Delta e_{\mathcal{M}} \simeq e_{\mathcal{M}}$ has been attributed to the chaotic nature of the physical system (Laskar 1989; Varadi et al. 2003; Batygin \& Laughlin 2008). However, it is not clear why the system's chaotic behavior starts dominating $e_{\mathcal{M}}$ 's evolution after e.g. $\sim 60 \mathrm{Myr}$ in mercury6 with bodycentric coordinates (Fig. 5a) but only after $\sim 120 \mathrm{Myr}$ in HNBody with Jacobi coordinates (Fig. 5c). The initial $\Delta e_{\mathcal{M}}(t=0)$ in both the mercury 6 and HNBody simulations was $\sim 3 \times 10^{-11}$. The time evolution of the absolute $\Delta e_{\mathcal{M}} \max -$ ima of the two curves (Fig. 5a,c) may be fit to simple functions assuming linear and exponential growth of the initial $\Delta e_{\mathcal{M}}$ (Fig. 6). The exponential function fits the rapid growth phase well in the mercury6 simulations (Body-Body) with an estimated Lyapunov time $\tau \simeq 3.2 \mathrm{Myr}$. However, the exponential function is a poor fit to the HNBody simulations (Jacobi-Jacobi) with an estimated Lyapunov time $\tau \simeq 6$ Myr (Fig. 6).

Nevertheless, these estimated Lyapunov times are in good agreement with estimates of Lyapunov exponents from phase space separation of two nearby orbits over time (Fig. 7). Note that strictly, Lyapunov exponents are derived from the solution of the variational equations, rather than from the evolution of two system trajectories (e.g. Holman \& Murray 1996; Tancredi et al. 2001; Morbidelli 2002). However, the emphasis here is on the relative difference between simulations with, say, different integration coordinates, while applying the same method for estimating Lyapunov exponents. Importantly, absolute estimates obtained here are consistent with results from previous studies (Laskar 1989; Varadi et al. 2003; Batygin \& Laughlin 2008). 

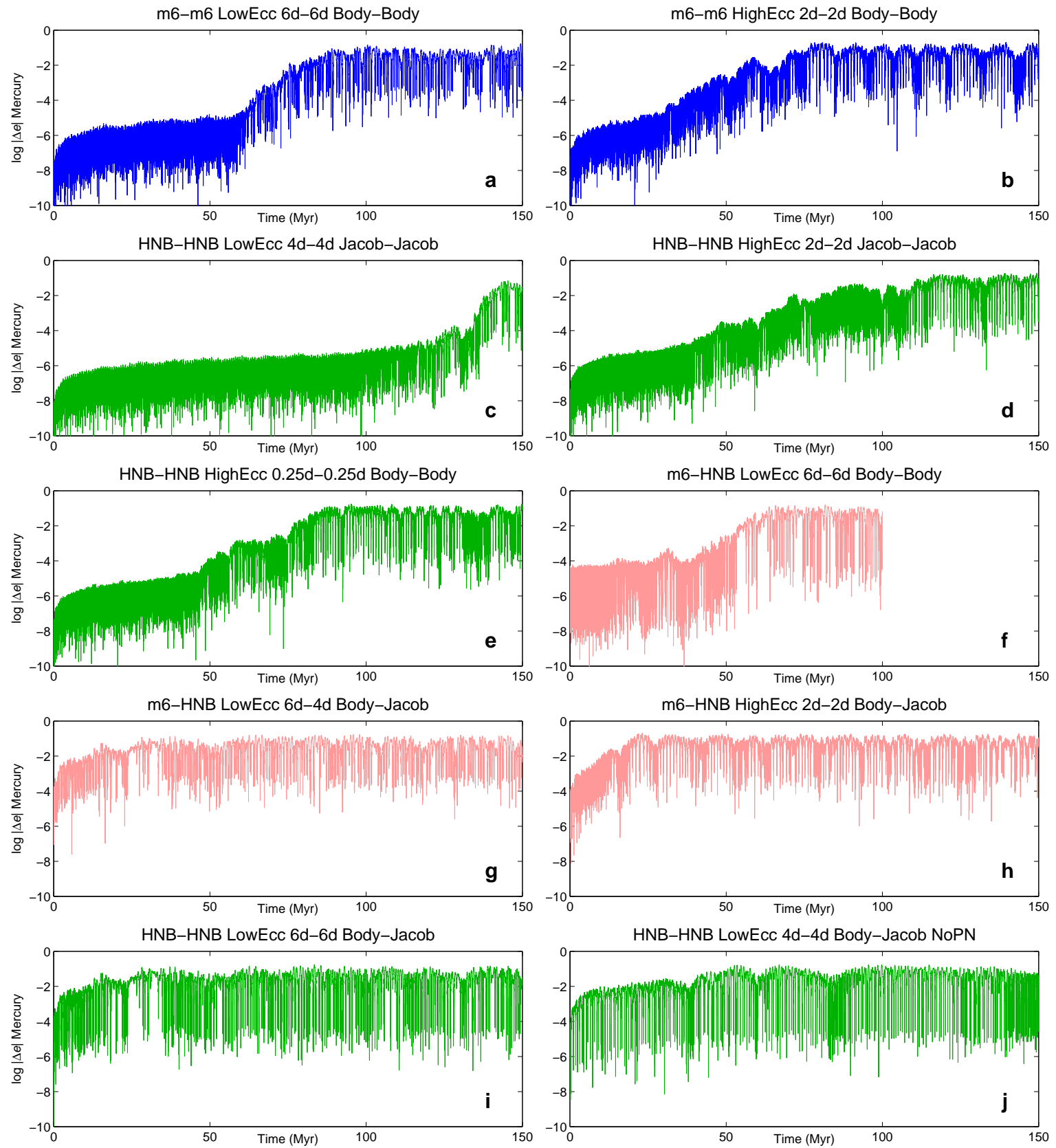

Fig. 5.- Computed difference in Mercury's eccentricity $\left(\Delta e_{\mathcal{M}}\right)$ between two runs each per panel for different integrations and options. Blue curves: both runs with mercury6 (m6), green curves: both runs with HNBody (HNB), light red curves: one run with mercury6, the other with HNBody. Body: bodycentric, Jacob: Jacobi coordinates. $m$ d- $n$ d indicates the timestep in both runs (in days). LowEcc/HighEcc: initial $e_{\mathcal{M}}=0.21 / 0.53$ (see text). NoPN: No contributions from general relativity. Panels a-e show differences in $e_{\mathcal{M}}$ between solutions with slightly different initial conditions ( $2.73 \mathrm{~m}$ offset in Mercury's initial radial distance); panels $\mathrm{f}-\mathrm{j}$ : identical initial conditions. Note rapid $\Delta e_{\mathcal{M}}$ rise in $\mathrm{g}-\mathrm{j}$ (all Body-Jacobi). 

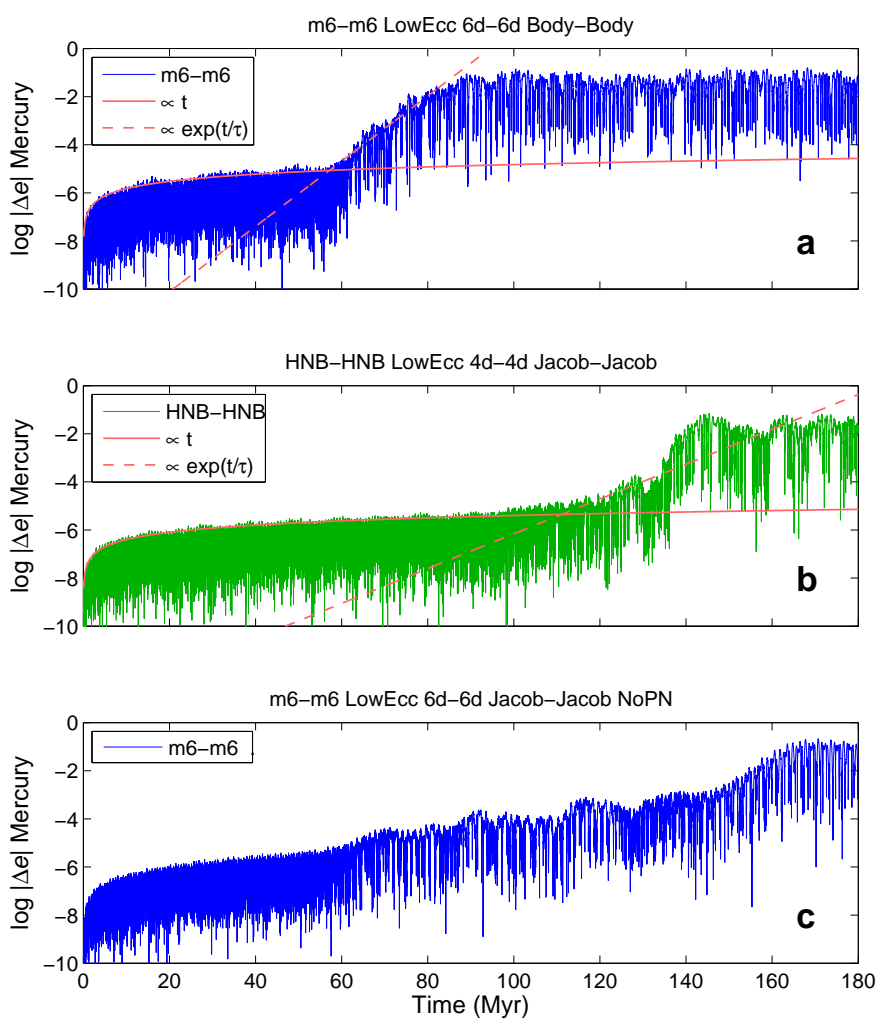

Fig. 6.- Computed difference in Mercury's eccentricity $\left(\Delta e_{\mathcal{M}}\right)$ between two runs each per panel with slightly different initial conditions ( $2.73 \mathrm{~m}$ offset in Mercury's initial radial distance) for different integrations and options. Blue curves: both runs with mercury6 (m6), green curve: both runs with HNBody (HNB), red curves: simple fit functions assuming linear and exponential growth of initial $\Delta e_{\mathcal{M}}$. Body: bodycentric, Jacob: Jacobi coordinates. $m \mathrm{~d}-n \mathrm{~d}$ indicates the timestep in both runs (in days). LowEcc: initial $e_{\mathcal{M}}=0.21$ (see text). NoPN: No contributions from general relativity. Note different slopes (estimated Lyapunov time $\tau$ ) of exponential fits in (a) and (b) (red dashed lines; linear on logarithmic y-scale).

The Lyapunov exponents were determined from two runs each with mercury6 and HNBody (one fiducial, one shadow orbit each) without renormalization and an initial separation of $5 \times 10^{-14} \mathrm{AU}$ in Mercury's $x$-coordinate. Renormalization was tested but yielded spurious results as reported before (Tancredi et al. 2001). If during each time interval $\delta t$, the distance in phase space grows exponentially, then $d_{j}=d_{j}^{0} \exp (\gamma \cdot \delta t)$, where $d_{j}^{0}$ and $d_{j}$ is the initial and final distance. An average $\gamma_{k}$ after $t=k \cdot \delta t$ may then be computed as:

$$
\gamma_{k}=\frac{1}{k \cdot \delta t} \sum_{j=1}^{k} \ln \left(d_{j} / d_{j}^{0}\right),
$$

where $\delta t$ was set to $20 \mathrm{kyr}$. A log-log plot of $\gamma_{k}$

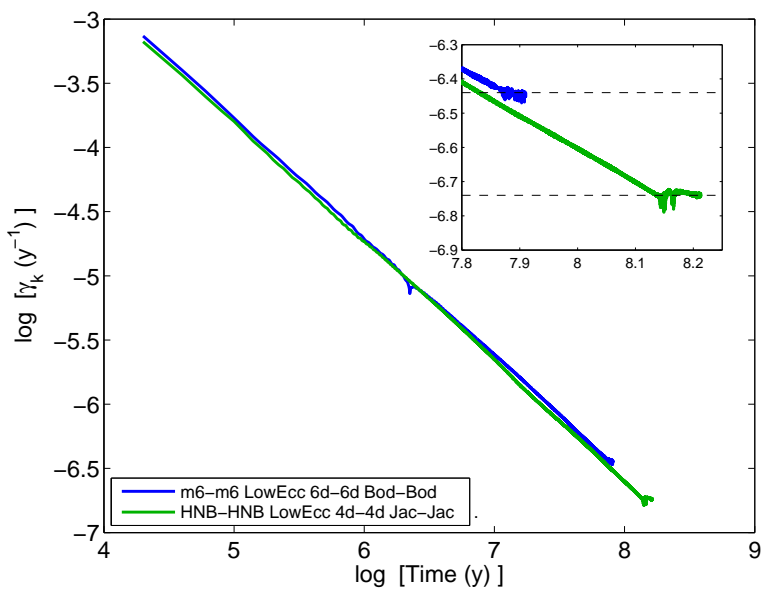

Fig. 7.- Lyapunov exponents estimated from phase space separation of two nearby orbits over time. Blue curve: both runs with mercury6 (m6), green curve: both runs with HNBody (HNB). Graphs have been truncated shortly after the inflection point (see text). Bod: bodycentric, Jac: Jacobi coordinates. $m \mathrm{~d}-n \mathrm{~d}$ indicates timestep in both runs (in days). LowEcc: initial $e_{\mathcal{M}}=0.21$.

vs. time yields a straight line with negative constant slope until an inflection point is reached after which $\gamma_{k}$ approaches a non-zero value (Fig. 7). The inflection point corresponds to the time in the integration where exponential growth starts dominating the evolution of the phase space distance between the two orbits. Note that the graphs in Fig. 7 have been truncated shortly after the inflection point to emphasize the plateau in $\gamma_{k}\left(\gamma_{k}\right.$ keeps decreasing subsequently as $d$ is bounded without renormalization, not shown). The corresponding Lyapunov exponents for the mercury6 and HNBody standard runs are $10^{-6.44}$ and $10^{-6.74}$ (i.e. Lyapunov times of $2.8 \mathrm{Myr}$ and $5.5 \mathrm{Myr}$ ), respectively, in good agreement with the Lyapunov times estimated from differences in Mercury's eccentricity evolution (Fig. 6). Note that based on different approaches it may be difficult to measure Lyapunov times with an absolute accuracy much better than a factor of two (Murray \& Holman 1999). However, based on the current approach (simulations with identical initial conditions, etc.), it is not difficult to measure Lyapunov times with a relative accuracy better than a factor of two (Figs. 6 and 7).

I also computed $\Delta e_{\mathcal{M}}$ over time for two simulations with slightly different initial conditions using Jacobi coordinates in mercury6 (mixed-variable symplectic algorithm) without contributions from 
general relativity (Fig. 6). In this case, selecting parameters for an exponential fit is not obvious but it is clear that it takes more than $160 \mathrm{Myr}$ for $\Delta e_{\mathcal{M}}$ to approach the magnitude of $e_{\mathcal{M}}$. The bottom line is that the estimated Lyapunov times for Mercury's orbit from the standard mercury 6 and HNBody integrations (Figs. 6 and 7) differ at least by a factor of two, which is easily measurable given the relative accuracy of the current approach. However, the chaotic behavior of the Solar System is a physical property that cannot depend on the numerical algorithm chosen to describe the system. Yet the results presented here suggest that an exponential divergence of trajectories starts dominating the numerical solutions after significantly different time intervals, depending on integration coordinates (Figs. 5, 6, 7). In addition, the rapid $\Delta e_{\mathcal{M}}$ growth over the first $10 \mathrm{Myr}$ between two solutions using the same initial conditions but different integration coordinates (Fig. $5 \mathrm{~g}-\mathrm{j}$ ) suggests a fundamental difference between algorithms using bodycentric and Jacobi coordinates.

\section{Algorithm tests}

The results presented above raise questions about numerical algorithm performance and the use of integration coordinates. Why do heliocentric and Jacobi coordinates lead to statistically different results? Which algorithm/integration coordinates provide more accurate results? This section describes several tests aiming at resolving the dilemma of the dependency of statistical results on integration coordinates.

\subsection{2-Body problem: Position errors}

One approach for testing the accuracy of a numerical algorithm is to study the 2-body problem, for which an analytical solution exist (strictly, Kepler's equation is solved numerically though). While substantially less complex than the general $n$-body problem, in the following the 2-body problem will illustrate one important difference between symplectic algorithms with heliocentric and Jacobi coordinates.

The Hamiltonian for the 2-body problem may be written as:

$$
H=\frac{\left|\boldsymbol{p}_{\mathbf{0}}\right|^{2}}{2 m_{0}}+\frac{\left|\boldsymbol{p}_{\mathbf{1}}\right|^{2}}{2 m_{1}}-\frac{G m_{0} m_{1}}{\left|\boldsymbol{x}_{0}-\boldsymbol{x}_{1}\right|},
$$

where $G$ is the gravitational constant and $\boldsymbol{p}_{i}, m_{i}$, and $\boldsymbol{x}_{i}$ are the momenta, masses, and positions of the two bodies. The problem can be simplified via canonical transformations, using e.g. democraticheliocentric (DH) and Jacobi coordinates, denoted here as:

$$
\begin{array}{lll}
(\boldsymbol{x}, \boldsymbol{p}) & \longrightarrow(\mathcal{Q}, \mathcal{P}): & \text { Democratic-Heliocentric } \\
(\boldsymbol{x}, \boldsymbol{p}) & \longrightarrow(\boldsymbol{Q}, \boldsymbol{P}): & \text { Jacobi } .
\end{array}
$$

In DH coordinates, $\mathcal{\mathcal { Q }}_{0}$ is the position of the center of mass and $\mathcal{Q}_{1}$ is the heliocentric position of $m_{1}$. One may use a generating function $F_{3}(\mathcal{Q}, \boldsymbol{p})$ of the new positions and the old momenta (Duncan et al. 1998):

$$
\begin{aligned}
F_{3}= & -\boldsymbol{p}_{0}\left(\mathcal{Q}_{0}-\frac{m_{1}}{M} \mathcal{Q}_{1}\right) \\
& -\boldsymbol{p}_{1}\left(\mathcal{Q}_{0}-\frac{m_{1}}{M} \mathcal{Q}_{1}+\mathcal{Q}_{1}\right),
\end{aligned}
$$

where $M=m_{0}+m_{1}$. The relationship between old and new variables then is:

$$
\begin{aligned}
-\frac{\partial F_{3}}{\partial \boldsymbol{p}_{0}} & =\boldsymbol{x}_{0}=\left(\mathcal{Q}_{0}-\frac{m_{1}}{M} \mathcal{Q}_{1}\right) \\
-\frac{\partial F_{3}}{\partial \boldsymbol{p}_{1}} & =\boldsymbol{x}_{1}=\left(\mathcal{Q}_{0}-\frac{m_{1}}{M} \mathcal{Q}_{1}+\mathcal{Q}_{1}\right) \\
-\frac{\partial F_{3}}{\partial \mathcal{Q}_{0}} & =\mathcal{P}_{0}=\boldsymbol{p}_{0}+\boldsymbol{p}_{1} \\
-\frac{\partial F_{3}}{\partial \mathcal{Q}_{1}} & =\mathcal{P}_{1}=\boldsymbol{p}_{1}-\frac{m_{1}}{M}\left(\boldsymbol{p}_{0}+\boldsymbol{p}_{1}\right) .
\end{aligned}
$$

Note that $\mathcal{P}_{0}$ is the total momentum and $\mathcal{P}_{1}$ is the barycentric momentum of $m_{1}$. After some algebra, the Hamiltonian becomes:

$$
H=\left(\frac{\left|\mathcal{P}_{1}\right|^{2}}{2 m_{1}}-\frac{G m_{0} m_{1}}{\left|\mathcal{Q}_{1}\right|}\right)+\frac{\left|\mathcal{P}_{0}\right|^{2}}{2 M}+\frac{\left|\mathcal{P}_{1}\right|^{2}}{2 m_{0}} .
$$

The first two terms in parentheses represent the Kepler Hamiltonian. The third term represents the motion of the center of mass, which moves as a free particle and can be ignored. The last term needs to be integrated separately in symplectic algorithms with $\mathrm{DH}$ coordinates. This term is often denoted as $H_{\text {Sun }}$ because $-\mathcal{P}_{1}=$ $\boldsymbol{p}_{0}-\left(m_{0} / M\right)\left(\boldsymbol{p}_{0}+\boldsymbol{p}_{1}\right)$ is the barycentric momentum of the Sun.

For Jacobi coordinates, one may use a generating function $F_{2}(\boldsymbol{x}, \boldsymbol{P})$ of the old positions and the new momenta:

$$
F_{2}=\boldsymbol{P}_{0}\left(m_{0} \boldsymbol{x}_{0}+m_{1} \boldsymbol{x}_{1}\right) / M+\boldsymbol{P}_{1}\left(\boldsymbol{x}_{1}-\boldsymbol{x}_{0}\right),
$$




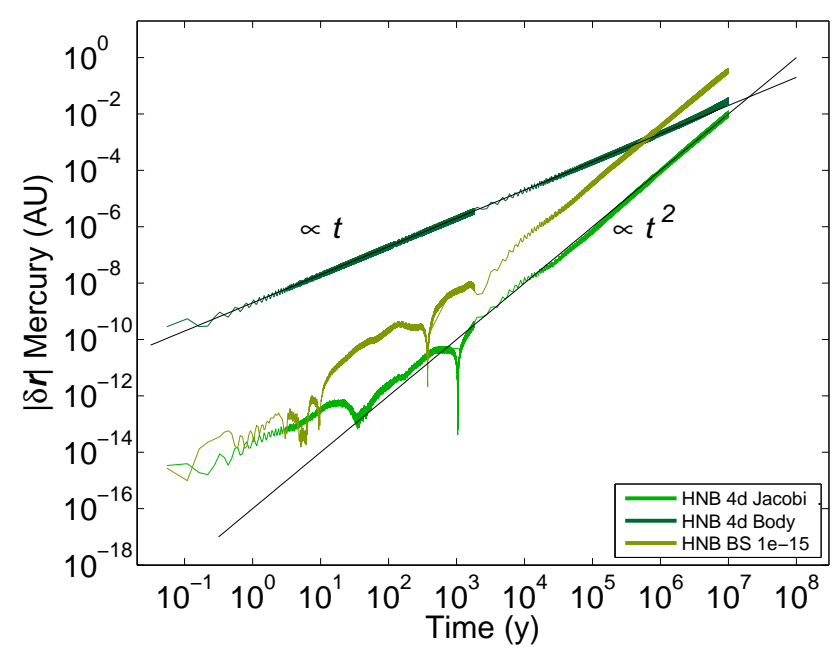

Fig. 8.- Difference between numerically and analytically determined position vector of Mercury $\left(\left|\delta \boldsymbol{r}_{\mathcal{M}}\right|=\left|\boldsymbol{r}_{\mathcal{M}}^{\text {num }}-\boldsymbol{r}_{\mathcal{M}}^{\text {anl }}\right|\right)$ from 2-body integrations with HNBody of the Sun and Mercury. Graphs labeled "Jacobi" and "Body" show $\left|\delta \boldsymbol{r}_{\mathcal{M}}\right|$ between the 2nd order symplectic integrator using Jacobi and bodycentric coordinates (both 4-day timestep) and the analytical solution. BS: $\left|\delta \boldsymbol{r}_{\mathcal{M}}\right|$ between non-symplectic Bulirsch-Stoer algorithm (relative accuracy set to $10^{-15}$ ) and analytical solution.

where $\left(m_{0} \boldsymbol{x}_{0}+m_{1} \boldsymbol{x}_{1}\right) / M=\boldsymbol{X}_{S}$ is the center of mass. The relationship between old and new variables then is:

$$
\begin{aligned}
& \frac{\partial F_{2}}{\partial \boldsymbol{P}_{0}}=\boldsymbol{X}_{S}=\boldsymbol{Q}_{0} \\
& \frac{\partial F_{2}}{\partial \boldsymbol{P}_{1}}=\left(\boldsymbol{x}_{1}-\boldsymbol{x}_{0}\right)=\boldsymbol{Q}_{1} \\
& \frac{\partial F_{2}}{\partial \boldsymbol{x}_{0}}=-\boldsymbol{P}_{1}+\boldsymbol{P}_{0} m_{0} / M=\boldsymbol{p}_{0} \\
& \frac{\partial F_{2}}{\partial \boldsymbol{x}_{1}}=\boldsymbol{P}_{1}+\boldsymbol{P}_{0} m_{1} / M=\boldsymbol{p}_{1}
\end{aligned}
$$

The Hamiltonian becomes:

$$
H=\left(\frac{\left|\boldsymbol{P}_{1}\right|^{2}}{2 \mu}-\frac{G m_{0} m_{1}}{\left|\boldsymbol{Q}_{1}\right|}\right)+\frac{\left|\boldsymbol{P}_{0}\right|^{2}}{2 M},
$$

where $\mu=m_{0} m_{1} / M$ is the reduced mass. Again, the first two terms represent the Kepler Hamiltonian and the third term (center of mass) can be ignored. However, comparing the Hamiltonian in Jacobi coordinates (Eq. (9)) and in DH coordinates (Eq. (7)) shows that in Jacobi coordinates the relevant Kepler mass is $\mu$ (rather than $m_{1}$ ) and $H_{\text {Sun }}$ is absent.

Thus, even for the simple 2-body problem, there is a difference between symplectic algorithms with heliocentric and Jacobi coordinates. Because the Hamiltonian is purely Keplerian in Jacobi coordinates (no solar correction), symplectic integration of the 2-body problem in Jacobi coordinates should be more accurate than in $\mathrm{DH}$ coordinates. Indeed, 10-Myr integrations with HNBody of the Sun and Mercury showed that $\left|\delta \boldsymbol{r}_{\mathcal{M}}\right|$ (difference in Mercury's numerical and analytical position vector) was substantially smaller in integrations with Jacobi than DH coordinates over the first 1 Myr or so (Fig. 8). On a timescale of $10^{-1} \mathrm{y}$, the numerical position error is close to machine precision for Jacobi coordinates but $\sim 10^{5}$-times larger for $\mathrm{DH}$ coordinates (both 4-day timestep). Subsequently, the position error grows approximately quadratic and linear in time for Jacobi and DH coordinates, respectively. Additional runs with HNBody using a non-symplectic Bulirsch-Stoer algorithm (relative accuracy set to $10^{-15}$ ) yields errors in $\left|\boldsymbol{r}_{\mathcal{M}}\right|$ closer to those of the symplectic integrator with Jacobi coordinates (Fig. 8). Note, however, that on timescales of $10^{6}-10^{7}$ y errors in $\left|\boldsymbol{r}_{\mathcal{M}}\right|$ grow to similarly large values in all three integrations.

Given that increases in Mercury's eccentricity are critical for the potential destabilization of the Solar System, accurate numerical integration of its orbit is key. Because Mercury is the innermost planet, one might argue that Jacobi coordinates are better suited than DH coordinates for this task (Duncan et al. 1998) and that integrating Solar System orbits (specifically Mercury's orbit) using DH coordinates simply lacks the necessary accuracy. In terms of errors in Mercury's position vector, the present 2-body integrations support this notion, but only on timescales shorter than $\sim 10^{6} \mathrm{y}$.

\subsection{Bulirsch-Stoer algorithm: Eccentric- ity errors}

As described above, I also tested whether the statistically different $e_{\mathcal{M}}^{\max }$ in the 500-Myr runs (high initial $e_{\mathcal{M}}$ ) are related to typically larger errors associated with bodycentric vs. Jacobi coordinates at the same step size. I repeated the 500Myr runs with the bodycentric HNBody setup but an eight-fold smaller timestep $(0.25 \mathrm{~d}$, 100-fold smaller $|\Delta E / E|)$. The basic result remained the same. At high initial $e_{\mathcal{M}}$, the setup using bodycentric coordinates leads to significantly smaller mean $e_{\mathcal{M}}^{\max }$ than the setup using Jacobi coordinates 
(Fig. 2). This result suggests that the effect of different integration coordinates on the statistics of Mercury's eccentricity evolution are not caused by errors arising from a too large timestep in the bodycentric setup.

Further tests can be performed by comparing the results of the symplectic integrators to results of non-symplectic integrators, e.g. the BulirschStoer (BS) algorithm. Note that such tests are usually run over shorter time intervals as in most cases non-symplectic integrations are computationally much more expensive than symplectic integrations. Comparison between HNBody's BS and the 2nd order symplectic integration of the eight planets and Pluto starting at initial $e_{\mathcal{M}}=0.53$ showed a moderate rise in $\Delta e_{\mathcal{M}}$ over $10 \mathrm{Myr}$ when using Jacobi coordinates and a 2-day timestep $\left(\max \left|\Delta e_{\mathcal{M}}\right| \lesssim 10^{-4}\right.$, Fig. 9a). This was not the case for bodycentric coordinates/2-day timestep vs. BS, where $\max \left|\Delta e_{\mathcal{M}}\right|$ grew rapidly to $\sim 10^{-2}$ over 10 Myr (Fig. 9b). However, a moderate rise was found again in $\Delta e_{\mathcal{M}}$ over $10 \mathrm{Myr}$ when using bodycentric coordinates and a 0.25-day timestep $\left(\max \left|\Delta e_{\mathcal{M}}\right| \lesssim 10^{-4}\right.$, Fig. 9c). Thus, in seeming contradiction to the results of the 500-Myr runs (see above), the comparison to BS suggests that differences in $e_{\mathcal{M}}$ may be rectified provided that the timestep in the bodycentric setup is "sufficiently small". If so, then what is sufficiently small?

For the symplectic 2-body integrations discussed above, a timestep smaller than $\sim 0.05$ days would achieve roughly the same accuracy with bodycentric coordinates as with Jacobi coordinates and a 4-day timestep. If the same timestep ratio were to apply to full Solar System integrations, then the timestep would have to be reduced by a factor of 80 when using bodycentric coordinates instead of Jacobi coordinates. For example, the 500-Myr runs with 2-day timestep ran over roughly 2.2 days wall-clock time. Thus, repeating those runs with an 80-times reduced timestep would take about 180 days (2.6 years for the 5 -Gyr runs). Not only are such runs currently impractical, at small timesteps one also needs to consider accumulation of numerical errors, which typically scale with the number of steps. Finally, could the accuracy of symplectic integrations be tested by comparison to long-term integrations using nonsymplectic algorithms such as Bulirsch-Stoer? As
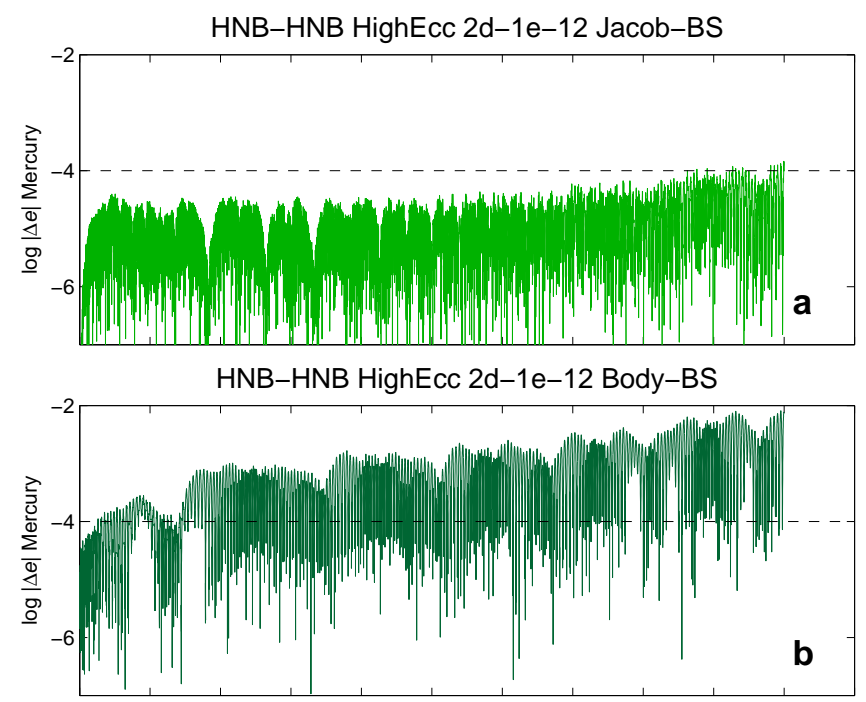

HNB-HNB HighEcc 0.25d-1e-12 Body-BS

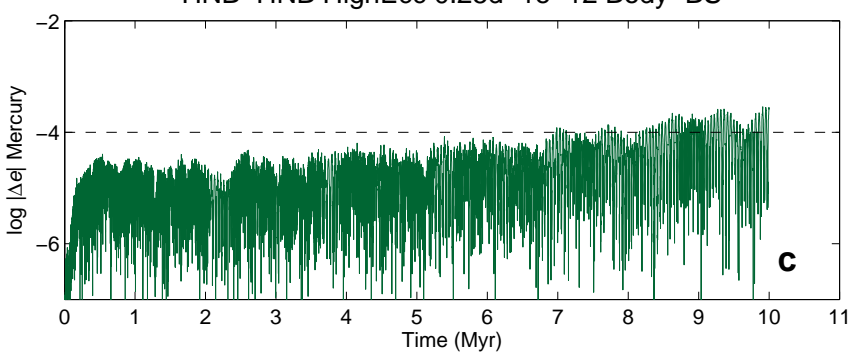

Fig. 9.- Computed difference in Mercury's eccentricity $\left(\Delta e_{\mathcal{M}}\right)$ between two runs each per panel for different integrations and options. All runs with HNBody (HNB) including the eight planets and Pluto. Body: bodycentric, Jacob: Jacobi coordinates, BS: Bulirsch-Stoer. HighEcc: initial $e_{\mathcal{M}}=0.53$ (see text). (a) 2nd order symplectic algorithm with 2-day timestep and Jacobi coordinates vs. non-symplectic Bulirsch-Stoer algorithm (relative accuracy set to $10^{-12}$ ). (b) Same as (a) but bodycentric coordinates. (c) Same as (b) but 0.25-day timestep.

mentioned above, the Bulirsch-Stoer algorithm is currently computationally too expensive for longterm integrations. In addition, non-symplectic algorithms usually suffer from substantial long-term drifts in total energy and angular momentum.

\subsection{Sun +5 planets: Statistics at high $e_{\mathcal{M}}$}

While long-term integrations of the full equations of motion of the complete Solar System at very small timesteps appear impractical at this stage, insight into algorithm performance may be gained from test integrations of planetary systems of somewhat reduced complexity. It turned out that the $\left(g_{1}-g_{5}\right)$ resonance pattern at high initial $e_{\mathcal{M}}$ can be reproduced with just a 6-body setup 


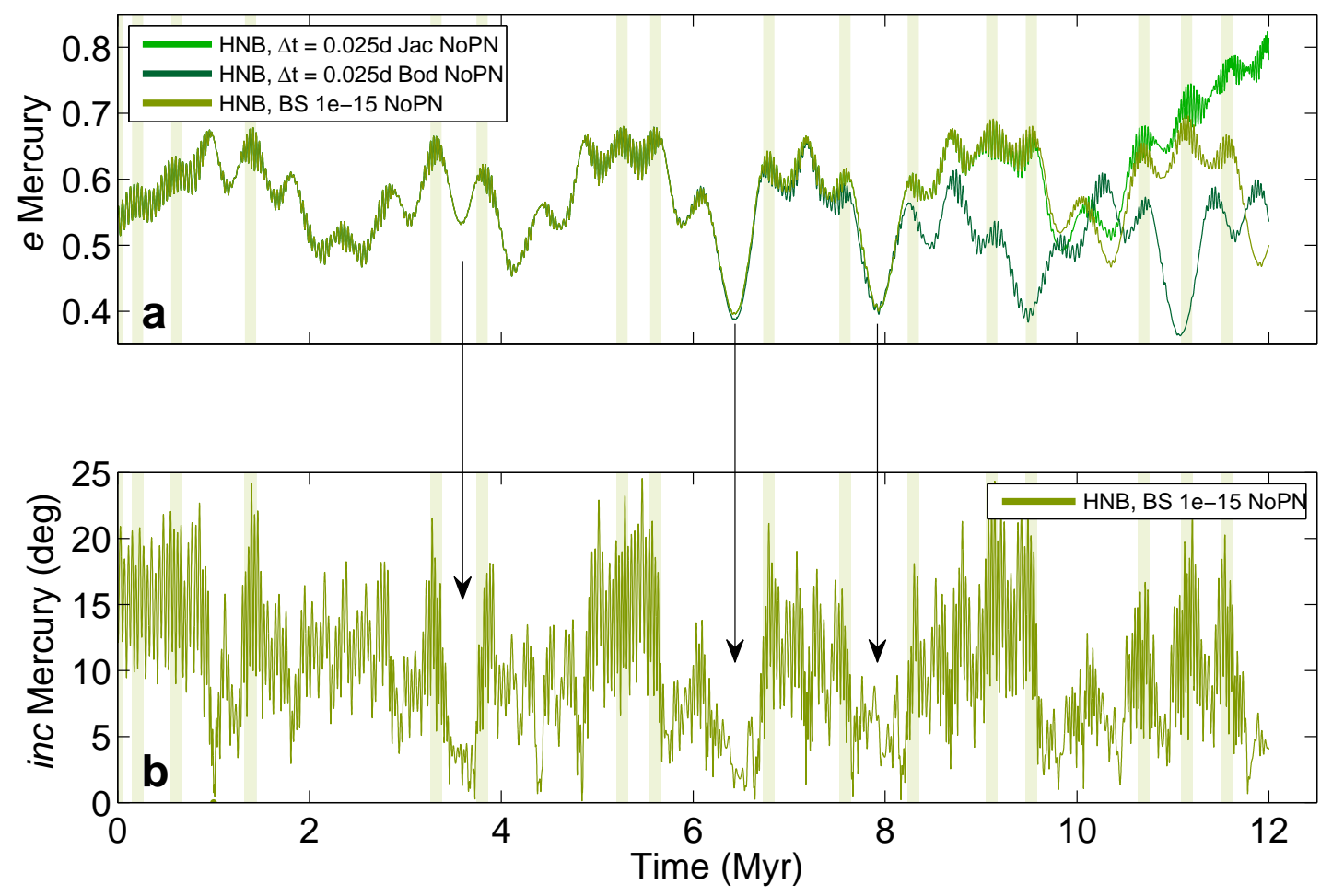

(Sun +5 planets: Mercury, Venus, Earth, Jupiter, and Saturn). When contributions from general relativity are ignored, this setup frequently leads to rapid $e_{\mathcal{M}}$ increases after $\sim 10 \mathrm{Myr}$. The estimated Lyapunov time for Mercury's orbit in this system is 0.6 Myr. Mercury's orbit is unstable and intermittently switches between resonant and nonresonant phases (Fig. 10). The resonant phase is associated with the $\left(g_{1}-g_{5}\right)$ resonance and typically high values in Mercury's inclination (ca. $10^{\circ}-$ $20^{\circ}$ ). The non-resonant phase is typically associated with a drop in Mercury's inclination and eccentricity. After about 8-10 Myr, $\Delta e_{\mathcal{M}}$ between two nearby orbits reaches the magnitude of $e_{\mathcal{M}}$ itself, which subsequently increases beyond 0.8 between 10-12 Myr in some simulation but not in others, depending (sensitively) on initial conditions. Hence, the system may provide some useful algorithm tests and allow statistical analyses over only a 12-Myr interval - an interval short enough for integrations with a very small timestep.

I have integrated 40 solutions each with four different numerical setups over $12 \mathrm{Myr}$ using HNBody (Fig. 11): (a) symplectic algorithm with 2-day timestep and Jacobi coordinates, (b) symplectic
Fig. 10.- Mercury's eccentricity and inclination from 12Myr runs (Sun +5 planets) at high initial $e_{\mathcal{M}}$ without contributions from general relativity $(\mathrm{NoPN})$. Jac: Jacobi, Bod: bodycentric, coordinates, $\Delta t$ : step size in days, BS: BulirschStoer (relative accuracy set to $10^{-15}$ ). (a) One example from each of three setups (see legend, total \# of runs for each setup $=40)$. (b) Mercury's inclination for the BS solution corresponding to (a). Vertical bars highlight several eccentricity maxima and high inclination values associated with the $\left(g_{1}-g_{5}\right)$ resonance (BS solution). Arrows indicate a few examples of eccentricity minima and low inclination values associated with non-resonant phases.

algorithm with 0.025-day timestep and bodycentric coordinates, (c) symplectic algorithm with 0.025-day timestep and Jacobi coordinates, (d) Bulirsch-Stoer (BS) algorithm (relative accuracy set to $10^{-15}$ ). All simulations ignore contributions from general relativity; the symplectic integrators are 2 nd order schemes. The setups (b) and (c) used an exact timestep of $0.025390625 \mathrm{~d}$ (finite binary representation to minimize round-off errors), which is about 80 times smaller than the 2-day timestep of setup (a) (see discussion Section 6.2). The four sets of integrations $(N=40$ each) started from the same set of initial conditions, where Mercury's initial radial distance was offset by $10 \mathrm{~m}$ between every two adjacent orbits (largest overall offset: $39 \times 10 \mathrm{~m}=390 \mathrm{~m}$ ). 

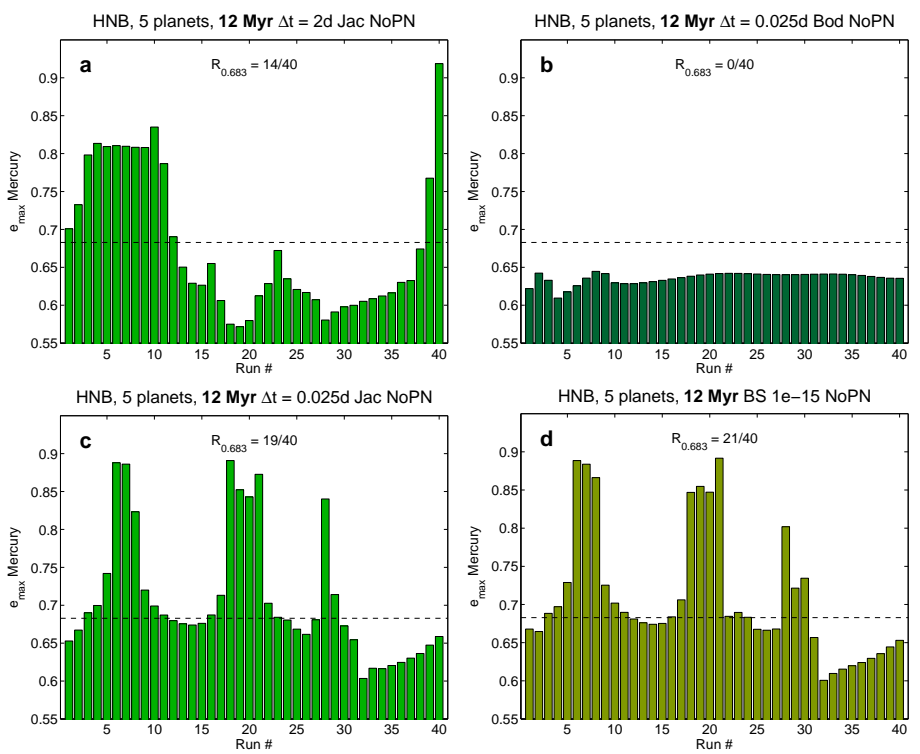

Fig. 11. - Mercury's maximum eccentricity $\left(e_{\mathcal{M}}^{\max }\right)$ achieved during final 2 Myr of $12-\mathrm{Myr}$ runs (Sun +5 planets, see text). Jac: Jacobi, Bod: bodycentric, coordinates, $\Delta t$ : step size in days, BS: Bulirsch-Stoer (relative accuracy set to $10^{-15}$ ), NoPN: No contributions from general relativity. $R_{0.683}$ indicates the number of solutions in which $e_{\mathcal{M}}$ grows beyond 0.683 (mean of all 160 runs, dashed lines).

For all simulations, Mercury's maximum eccentricity $\left(e_{\mathcal{M}}^{\max }\right)$ achieved during the final 2 Myr of the 12-Myr runs was recorded, providing a metric for either an increase or a decline in $e_{\mathcal{M}}^{\max }$ towards the end of the integration (Fig. 10). Of the four numerical setups, only the symplectic integrator with $\Delta t=0.025 \mathrm{~d} /$ Jacobi coordinates and the Bulirsch-Stoer algorithm yield similar statistical results for $e_{\mathcal{M}}^{\max }$ (Fig. 11). In comparison, the symplectic integrator with $\Delta t=2 \mathrm{~d} /$ Jacobi coordinates shows a reduced tendency for large $e_{\mathcal{M}}$ increases and has a lower mean $e_{\mathcal{M}}^{\max }$ value. Strikingly, the symplectic integrator with bodycentric coordinates predicts a decline in $e_{\mathcal{M}}^{\max }$ to values below 0.65 between 10-12 Myr in all simulations (0.65 is roughly equal to the $e_{\mathcal{M}}^{\max }$ value from 0 10 Myr, see Fig. 10).

For this particular system, the statistical results shown in Fig. 10 have important implications. Based on the agreement between BS and the symplectic integrations with $\Delta t=0.025 \mathrm{~d} / \mathrm{Jacobi}$ coordinates, it is likely that these two setups provide the most accurate results. The first implication then is that $\Delta t=2 \mathrm{~d}$ is too large for a 2nd order symplectic integrator with Jacobi coor- dinates at high $e_{\mathcal{M}}$. For instance, the calculated odds for $e_{\mathcal{M}}^{\max }$ to increase beyond 0.683 (mean of all 160 runs) are $48 \%$ at $\Delta t=0.025 \mathrm{~d}$ but only $35 \%$ at $\Delta t=2 \mathrm{~d}$. Second, the symplectic integrator with bodycentric coordinates yields incorrect results for this system even at a very small time step of $0.025 \mathrm{~d}=36 \mathrm{~min}$ ! The tendency to underestimate increases in Mercury's eccentricity at high $e_{\mathcal{M}}$, which was also observed in the 500-Myr integrations of the full Solar System (Fig. 5), is therefore unlikely related to errors associated with the size of the timestep. Fundamental differences in the implementation of different integration coordinates in symplectic schemes are more likely to be the cause (Section 6.1).

The results of the 12-Myr runs also help resolving the apparent contradiction between the 500Myr statistics and the $e_{\mathcal{M}}$ comparison to BS mentioned in Section 6.2. On the one hand, the 500Myr results suggest that the different $e_{\mathcal{M}}^{\max }$ statistics are largely independent of the step size in the bodycentric setup (Fig. 2). On the other hand, the comparison to BS suggests that differences in $e_{\mathcal{M}}$ can be minimized when the step size in the bodycentric setup is reduced (Fig. 9). In fact, for the 12-Myr runs both statements are correct. At a small timestep of $0.025 \mathrm{~d}, \Delta e_{\mathcal{M}}$ (BS minus Jacobi setup) and $\Delta e_{\mathcal{M}}$ (BS minus bodycentric setup) are virtually identical during the first $4 \mathrm{Myr}$ (not shown). In this interval, $\Delta e_{\mathcal{M}}$ is dominated by polynomial growth. During the final $2 \mathrm{Myr}$, however, all runs of the bodycentric setup show a decline in $e_{\mathcal{M}}^{\max }$ (contrary to the BS and Jacobi setup). In this interval, $\Delta e_{\mathcal{M}}$ is dominated by exponential growth. Importantly, the final interval determines the statistics of Mercury's ultimate eccentricity evolution. Thus, a smaller step size does improve the bodycentric setup's accuracy during polynomial growth of $\Delta e_{\mathcal{M}}$ but has little effect during exponential growth of $\Delta e_{\mathcal{M}}$.

Note that the implications outlined above strictly only apply to the system of reduced complexity studied in this section. That is, a system comprising the Sun and just five planets with specific initial conditions (including high initial $e_{\mathcal{M}}$ ), integrated over only $12 \mathrm{Myr}$ and ignoring PostNewtonian corrections. 


\section{Conclusions}

Reliable predictions of the Solar System's dynamic stability over billions of years not only require integrators that are fast and accurate but also produce robust statistical results in ensemble integrations. Ensemble integrations are necessary because of the chaotic behavior of the system. Currently, symplectic integrators are probably the best, if not the only, choice in terms of speed and accuracy. However, the present results show that tackling statistics is trickier as, for instance, the predicted probability for a large increase in Mercury's eccentricity $\left(e_{\mathcal{M}}\right)$ depends on the choice of integration coordinates in symplectic algorithms. Several tests performed here suggest that using Jacobi coordinates in symplectic integrations of the Solar System is more reliable than using bodycentric coordinates when Mercury's orbital eccentricity is high. However, these tests reveal little about the statistics of long-term integrations when Mercury's initial orbital eccentricity is low. In fact, the influence of Jacobi and bodycentric coordinates on the statistics of Mercury's eccentricity evolution appears to be opposite over 5 Gyr at low initial $e_{\mathcal{M}}$ than over $500 \mathrm{Myr}$ at high initial $e_{\mathcal{M}}$. Moreover, even accepting superiority of Jacobi coordinates at high $e_{\mathcal{M}}$, what is the proper step size to obtain robust statistical results? If applicable, the results of the system of reduced complexity (Section 6.3) suggest a timestep that could be as small as $0.025 \mathrm{~d}$. Even when starting with a larger timestep and reducing the timestep during the symplectic integration (which should be avoided), such small timesteps would still pose a challenge in terms of integration time. Then do symplectic integrations with Jacobi coordinates and typical timesteps of several days underestimate the odds for disaster (Fig. 11)? Again, if applicable, such simulations would underestimate the odds for disaster once $e_{\mathcal{M}}$ has already reached values $>0.5$ or so. But it is not clear whether the odds are predicted correctly to reach those values in the first place when starting at low $e_{\mathcal{M}}$.

It appears that several centuries of analytical research, modern state-of-the-art numerical algorithms, and current CPU power has brought us closer to answering the question of the Solar System's long-term stability. However, a definite, robust answer still seems to be lacking, including an- swers based on statistical approaches. It is likely that the odds for the catastrophic destabilization of the inner planets are in the order of a few percent. But what percentage exactly?

Acknowledgments. I thank the anonymous reviewer for constructive comments and Don David Ho and B. R. Oppenheimer for helpful conversations. I am grateful to Peter H. Richter who dared to introduce us to Chaos, Poincaré, and solar system dynamics in a 1989-undergraduate physics course on classical mechanics. 


\section{A. Contributions from general relativity}

Relativistic corrections are critical as they substantially reduce the probability for Mercury's orbit to achieve large eccentricities (Laskar \& Gastineau 2009). General relativity (GR) corrections are available in HNBody but not in mercury6. Post-Newtonian (PN) corrections for symplectic integration (Mikkola 1998; Soffel 1989) were therefore implemented before using mercury6. For more information on symplectic algorithms, see Wisdom \& Holman (1991); Saha \& Tremaine (1992); Chambers (1999). Note that the PN code was fully embedded in the symplectic hybrid integrator mdt_hy(), and not just added as an auxiliary force term in mfo_user(). For every planet, one needs to solve an equation of the form (Mikkola 1998):

$$
\boldsymbol{a}=\ddot{\boldsymbol{r}}=\boldsymbol{F}+\boldsymbol{f}_{0}(\boldsymbol{r}, t)+\frac{1}{c^{2}} \boldsymbol{f}_{1}(\boldsymbol{r}, \boldsymbol{v}),
$$

where $\boldsymbol{F}$ is the two-body acceleration, $\boldsymbol{f}_{0}(\boldsymbol{r}, t)$ is the Hamiltonian perturbation, $c$ is the speed of light, and $\boldsymbol{f}_{1}(\boldsymbol{r}, \boldsymbol{v})$ is the expression for the first Post-Newtonian contribution (Soffel 1989). Combining the implicit midpoint method and generalized leapfrog, and denoting the timestep as $h$, one obtains for the velocity jumps (Mikkola 1998):

$$
\delta \boldsymbol{v}=h \boldsymbol{f}_{0}(\boldsymbol{r}, t)+\frac{h}{c^{2}} \boldsymbol{f}_{1}\left(\boldsymbol{r}, \boldsymbol{v}+\frac{1}{2} \delta \boldsymbol{v}\right),
$$

which must be solved for $\delta \boldsymbol{v}$. The first PN acceleration term may be written as (Soffel 1989):

$$
\frac{1}{c^{2}} \boldsymbol{f}_{1}(\boldsymbol{r}, \boldsymbol{v})=\frac{G M}{c^{2}}\left[-\frac{\boldsymbol{v}^{2}}{r^{3}} \boldsymbol{r}+4 \frac{G M}{r^{4}} \boldsymbol{r}+4 \frac{(\boldsymbol{r} \cdot \boldsymbol{v})}{r^{3}} \boldsymbol{v}\right]
$$

Inserting into Eq. (A2) yields:

$$
\begin{aligned}
\delta \boldsymbol{v}= & h \boldsymbol{f}_{0}(\boldsymbol{r}, t) \\
& +h \frac{G M}{c^{2}}\left[-\frac{\left(\boldsymbol{v}+\frac{1}{2} \delta \boldsymbol{v}\right)^{2}}{r^{3}} \boldsymbol{r}+4 \frac{G M}{r^{4}} \boldsymbol{r}+4 \frac{\left[\boldsymbol{r} \cdot\left(\boldsymbol{v}+\frac{1}{2} \delta \boldsymbol{v}\right)\right]}{r^{3}}\left(\boldsymbol{v}+\frac{1}{2} \delta \boldsymbol{v}\right)\right],
\end{aligned}
$$

which was solved iteratively for $\delta \boldsymbol{v}$. Because the relativistic term is small, convergence is rapid (usually 2 iterations), and $\left[\delta \boldsymbol{v}(i=2)-\delta \boldsymbol{v}_{0}\right] / \delta \boldsymbol{v}_{0}<5 \times 10^{-16}$ for Mercury. The computational overhead for including GR contributions in mercury6 as described above was about 15-20\% (wall-clock time). Over the 21st century, Mercury's average perihelion precession (only due to GR) was 0.42976 " $\mathrm{y}^{-1}$ computed with HNBody and $0.42978 " \mathrm{y}^{-1}$ computed with mercury6 and the above GR implementation. In terms of Mercury's longterm eccentricity $\left(e_{\mathcal{M}}\right)$ evolution, the difference in $e_{\mathcal{M}}$ between mercury6 and HNBody runs (both with GR correction, $\Delta t=6$ days, and bodycentric coordinates) was $\sim 10^{-4}$ over the first $40 \mathrm{Myr}$. 


\section{REFERENCES}

Batygin, K., \& Laughlin, G. 2008, Astrophys. J., 683,1207

Boué, G., Laskar, J., \& Farago, F. 2012, Astron. Astroph., 548, A43

Chambers, J. E. 1999, Mon. Not. R. Astron. Soc., 304, 793

Duncan, M. J., Levison, H. F., \& Lee, M. H. 1998, Astron. J., 116, 2067

Einstein, A. 1916, Annalen der Physik, VI. Folge, $49(7), 769$

Farrés, A., Laskar, J., Blanes, S., et al. 2013, Celest. Mech. Dyn. Astr., 116, 141

Holman, M. J., \& Murray, N. W. 1996, Astron. J., 112(3), 1278

Ito, T., \& Tanikawa, K. 2002, MNRAS, 336, 483

Laplace, P. S. 1951, in (Dover Publications, New York)

Laskar, J. 1989, Nature, 338, 237

—. 2008, Icarus, 196, 1

Laskar, J. 2013, in Progress in Mathematical Physics, Vol. 66, Chaos, ed. B. Duplantier, S. Nonnenmacher, \& V. Rivasseau (Springer Basel), 239-270

Laskar, J., \& Gastineau, M. 2009, Nature, 459, 817

Lithwick, Y., \& Wu, Y. 2011, Astrophys. J., 739, $17 \mathrm{pp}$.

Mikkola, S. 1998, Celest. Mech. Dyn. Astr., 68, 249

Morbidelli, A. 2002, Modern Celestial Mechanics: Aspects of Solar System Dynamics (Taylor \& Francis, London)

Murray, N., \& Holman, M. 1999, Science, 283, 1877

Quinn, T. R., Tremaine, S., \& Duncan, M. 1991, Astron. J., 101, 2287
Rauch, K. P., \& Hamilton, D. P. 2002, in Bull. Am. Astron. Soc., Vol. 34, AAS/Division of Dynamical Astronomy Meeting \#33, 938

Richter, P. H. 2001, in Reviews in Modern Astronomy: Dynamic Stability and Instabilities in the Universe, ed. R. E. Schielicke, Vol. 14, 53-92

Saha, P., \& Tremaine, S. 1992, Astron. J., 104, 1633

Soffel, M. H. 1989, Relativity in Astrometry, Celestial Mechanics and Geodesy (Springer-Verlag Heidelberg, pp. 208)

Sussman, G. J., \& Wisdom, J. 1992, Science, 257, 56

Tancredi, G., Sánchez, A., \& Roig, F. 2001, Astron. J., 121, 1171

Varadi, F., Runnegar, B., \& Ghil, M. 2003, Astrophys. J., 592, 620

Wisdom, J., \& Holman, M. 1991, Astron. J., 102, 1528

Yoshida, H. 1993, Celest. Mech. Dyn. Astron., 56, 27

This 2-column preprint was prepared with the AAS LATEX macros v5.2. 
Table 2: Initial conditions of the eight planets and Pluto $^{a}$ for 5-Gyr runs from DE431. Heliocentric positions $\boldsymbol{r}$ (AU) and velocities $\boldsymbol{v}\left(\mathrm{AU} \mathrm{d}^{-1}\right)$.

\begin{tabular}{|c|c|c|c|}
\hline & $x$ & $y$ & $z$ \\
\hline & & Mercury & \\
\hline$r$ & $-1.40712354144735680 \mathrm{E}-01$ & $-4.43906230277241465 \mathrm{E}-01$ & $-2.33474338281349329 \mathrm{E}-02$ \\
\hline \multirow[t]{2}{*}{$v$} & $+2.11691765462179472 \mathrm{E}-02$ & $-7.09701275933066148 \mathrm{E}-03$ & $-2.52278032052283448 \mathrm{E}-03$ \\
\hline & & Venus & \\
\hline$r$ & $-7.18629835259113170 \mathrm{E}-01$ & $-2.25188858612526514 \mathrm{E}-02$ & $+4.11716131772919824 \mathrm{E}-02$ \\
\hline \multirow[t]{2}{*}{$v$} & $+5.13955712094533914 \mathrm{E}-04$ & $-2.03061283748202266 \mathrm{E}-02$ & $-3.07198741951420558 \mathrm{E}-04$ \\
\hline & & Earth + Moon & \\
\hline$r$ & $-1.68563248623229384 \mathrm{E}-01$ & $+9.68761420122898564 \mathrm{E}-01$ & $-1.15183154209270563 \mathrm{E}-06$ \\
\hline \multirow[t]{2}{*}{$v$} & $-1.72299715055074729 \mathrm{E}-02$ & $-3.01349780674632205 \mathrm{E}-03$ & $+2.41254068070491868 \mathrm{E}-08$ \\
\hline & & Mars & \\
\hline$r$ & $+1.39036162161402177 \mathrm{E}+00$ & $-2.09984400533893799 \mathrm{E}-02$ & $-3.46177919349353047 \mathrm{E}-02$ \\
\hline \multirow[t]{2}{*}{$v$} & $+7.47813544105227729 \mathrm{E}-04$ & $+1.51863004086334515 \mathrm{E}-02$ & $+2.99756038504512547 \mathrm{E}-04$ \\
\hline & & Jupiter & \\
\hline$r$ & $+4.00345668418424960 \mathrm{E}+00$ & $+2.93535844833712467 \mathrm{E}+00$ & $-1.01823217020834328 \mathrm{E}-01$ \\
\hline \multirow[t]{2}{*}{$v$} & $-4.56348056882991196 \mathrm{E}-03$ & $+6.44675255807273997 \mathrm{E}-03$ & $+7.54565159392195741 \mathrm{E}-05$ \\
\hline & & Saturn & \\
\hline$r$ & $+6.40855153734800886 \mathrm{E}+00$ & $+6.56804703677062207 \mathrm{E}+00$ & $-3.69127809402511886 \mathrm{E}-01$ \\
\hline \multirow[t]{2}{*}{$v$} & $-4.29112154163879215 \mathrm{E}-03$ & $+3.89157880254167561 \mathrm{E}-03$ & $+1.02876894772680478 \mathrm{E}-04$ \\
\hline & & Uranus & \\
\hline$r$ & $+1.44305195077618524 \mathrm{E}+01$ & $-1.37356563056406209 \mathrm{E}+01$ & $-2.38128487167790809 \mathrm{E}-01$ \\
\hline \multirow[t]{2}{*}{$v$} & $+2.67837949019966498 \mathrm{E}-03$ & $+2.67244291355153403 \mathrm{E}-03$ & $-2.47764637737944378 \mathrm{E}-05$ \\
\hline & & Neptune & \\
\hline$r$ & $+1.68107582839480649 \mathrm{E}+01$ & $-2.49926499733276124 \mathrm{E}+01$ & $+1.27271208982211476 \mathrm{E}-01$ \\
\hline \multirow[t]{2}{*}{$v$} & $+2.57936917068014599 \mathrm{E}-03$ & $+1.77676956230748452 \mathrm{E}-03$ & $-9.59089132565213410 \mathrm{E}-05$ \\
\hline & & Pluto & \\
\hline$r$ & $-9.87686582399026491 \mathrm{E}+00$ & $-2.79580297772433077 \mathrm{E}+01$ & $+5.85080284687055574 \mathrm{E}+00$ \\
\hline$v$ & $+3.02870206449818878 \mathrm{E}-03$ & $-1.53793257901232473 \mathrm{E}-03$ & $-7.12171623386267461 \mathrm{E}-04$ \\
\hline
\end{tabular}

${ }^{a}$ Masses (Mercury to Pluto in solar masses): 1.66013679527193035E-07, 2.44783833966454472E-06, 3.04043264626852573E06, 3.22715144505387430E-07, 9.54791938424322164E-04, 2.85885980666102893E-04, 4.36625166899970042E-05, 5.15138902053549668E-05, 7.40740740740740710E-09. 